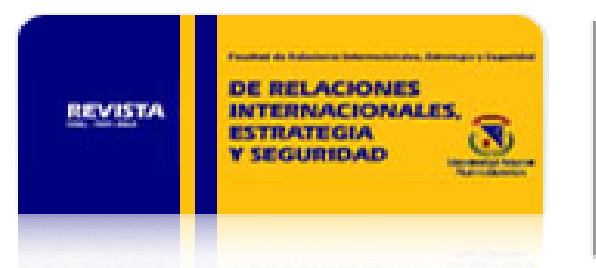

Revista de Relaciones Internacionales, Estrategia y Seguridad

ISSN: 1909-3063

cinuv.relinternal@unimilitar.edu.co

Universidad Militar Nueva Granada

Colombia

Picón Duarte, Ángela Milena

EL SURGIMIENTO DE NUEVOS DONANTES Y SUS IMPLICACIONES PARA LA ARQUITECTURA

DE LA AYUDA AL DESARROLLO

Revista de Relaciones Internacionales, Estrategia y Seguridad, vol. 4, núm. 1, enero-junio, 2009, pp. 69-88

Universidad Militar Nueva Granada

Bogotá, Colombia

Disponible en: http://www.redalyc.org/articulo.oa?id=92712970005

- Cómo citar el artículo

Número completo

- Más información del artículo

- Página de la revista en redalyc.org

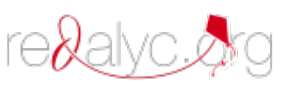

Sistema de Información Científica

Red de Revistas Científicas de América Latina, el Caribe, España y Portugal

Proyecto académico sin fines de lucro, desarrollado bajo la iniciativa de acceso abierto 
rev.relac.int.estrateg.segur.4(1):69-88,2009

\title{
EL SURGIMIENTO DE NUEVOS DONANTES Y SUS IMPLICACIONES PARA LA ARQUITECTURA DE LA AYUDA AL DESARROLLO
}

\author{
Ángela Milena Picón Duarte*
}

\section{RESUMEN}

Este documento analiza los posibles riesgos, beneficios y desafíos futuros para la arquitectura de ayuda al desarrollo, a partir de la emergencia de nuevos donantes no miembros del Comité de Ayuda al Desarrollo -CAD-. Para ello, en primer lugar, se hará referencia al surgimiento de economías emergentes y su configuración como nuevos donantes de ayuda al desarrollo. En segundo lugar, se identificarán los posibles riesgos y beneficios que conlleva la ayuda al desarrollo de estos nuevos donantes, con el propósito de analizar los futuros desafíos que deberá enfrentar la actual arquitectura de ayuda. Por último, se elaborarán algunas conclusiones y reflexiones finales.

Palabras clave: nuevos donantes, economías emergentes, CAD, ayuda al desarrollo, cooperación, objetivos del milenio.

* Politóloga, Pontificia Universidad Javeriana, Facultad de Ciencias Políticas y Relaciones Internacionales. Magíster en Diplomacia y Relaciones Internacionales, Escuela Diplomática de Madrid. anpicond@yahoo.com 


\begin{abstract}
This paper analyzes the risks, benefits and future challenges of the architecture of the development aid since the appearance of new donors others than those within the Development Assistant Committee-DAC-. For this purpose, first of all, it will briefly describe the appearance of the emerging economies and their configuration as new donors of development aid; secondly, it will identify some possible risks and benefits that the development aid of the new donors implies, in order to analyze the challenges that the current architecture of the development aid will have to face in the near future. Finally, it will present some conclusions.
\end{abstract}

Key words: new donors, emerging economies, DAC, development aid, cooperation, Millennium Development Goals.

\title{
INTRODUCCIÓN
}

El surgimiento de economías emergentes, es decir, aquellos países que tradicionalmente se han caracterizado por ser receptores de ayuda al desarrollo pero que recientemente, dado su importante crecimiento económico se han convertido en donantes de ayuda -, como es el caso de China, India, Brasil, Sudáfrica, Rusia, Indonesia, México y Turquía principalmente-, ha dado como resultado la configuración de nuevos donantes de ayuda al desarrollo. Esta realidad supone un factor de cambio y de redefinición de la arquitectura de la ayuda al desarrollo, originariamente coordinada por los países miembros del Comité de Ayuda al Desarrollo -CAD- ${ }^{1}$, al mismo tiempo que conlleva al surgimiento de nuevos desafíos para la política de ayuda en cuanto a la manera como ésta es financiada, dirigida y coordinada.

$Y$ aunque existen posiciones a favor en lo referente a beneficios y contribuciones que estos nuevos donantes podrían proporcionar a la ayuda al desarrollo, existen muchas dudas y preocupaciones sobre el impacto y los posibles riesgos que ésta podría tener tanto para la actual arquitectura de ayuda como para la política de cooperación al desarrollo. Este hecho ha llevado a los países miembros del CAD a dar los primeros pasos para lograr un mayor acercamiento y diálogo con estos nuevos donantes. Estos últimos, al encontrase por fuera del CAD, no sólo han respondido a lógicas e intereses particulares, privilegiando sus propios estándares y lineamientos en cuanto a cooperación al desarrollo se refiere, sino que también han contribuido con nuevas perspectivas y valores que podrían de alguna manera reorientar y perfilar de una forma más acertada la actual arquitectura de cooperación.

\footnotetext{
${ }^{1}$ El CAD es el principal órgano de la Organización para la Cooperación y el Desarrollo Económico -OCDE-, y se encarga de los asuntos que tienen que ver con la cooperación al desarrollo. Está conformado por 23 miembros: Alemania, Australia, Austria, Bélgica, Canadá, Comisión de las Comunidades Europeas, Dinamarca, España, Estados Unidos, Finlandia, Francia, Grecia, Irlanda, Italia, Japón, Luxemburgo, Noruega, Nueva Zelanda, Países Bajos, Portugal, Reino Unido, Suecia y Suiza. Participan como observadores permanentes: el Fondo Monetario Internacional -FMI-, el Banco Mundial y el Programa de Naciones Unidas para el Desarrollo -PNUD-. Ver: Development Cooperation Directorate-DCD-DAC-. «DAC Members and date of membership» En: http://www.oecd.org/document/ 38/0,3343,en_2649_34603_1893350_1_1_1_1,00.html. Última consulta, abril 1 de 2008.
}

EL SURGIMIENTO DE NUEVOS DONANTES Y SUS IMPLICACIONES PARA LA ARQUITECTURA DE LA AYUDA AL DESARROLLO 
Teniendo en cuenta esta realidad, este documento analizará los posibles riegos, beneficios y futuros desafíos para la arquitectura de ayuda al desarrollo a partir de la emergencia de nuevos donantes no miembros del CAD. Para ello, en primer lugar, se hará referencia al surgimiento de economías emergentes y su configuración como nuevos donantes de ayuda al desarrollo. Luego, se identificarán los posibles riesgos y beneficios que conlleva la ayuda al desarrollo de nuevos donantes, con el propósito de analizar los futuros desafíos que deberá enfrentar la actual arquitectura de ayuda. Por último, se elaborarán algunas conclusiones y reflexiones finales.

\section{ECONOMÍAS EMERGENTES Y SU CONFIGURACIÓN COMO NUEVOS DONANTES DE AYUDA AL DESARROLLO}

El reiterado crecimiento económico de países como China, India, Brasil, Sudáfrica, Rusia, Indonesia, México y Turquía supone un factor de reequilibrio de las relaciones de poder y de redistribución de la riqueza a nivel internacional. Estos países comienzan a ejercer presión y a tener un peso considerable en la economía y en la política, permitiéndoles de alguna manera reconfigurar el panorama internacional actual. Un claro ejemplo de ello es la creación del $\mathrm{G} 20^{2}$, en donde países como Brasil, India y Sudáfrica se han dotado de capacidades de veto del que antes sólo podía ejercer Estados Unidos y la Unión Europea en el ámbito de la Organización Mundial del Comercio-OMC-3.

En lo que respecta a la ayuda al desarrollo, estos países han comenzado a establecer sus propias políticas de cooperación respondiendo a intereses y a necesidades puntuales que, de alguna manera, los ha llevado a privilegiar unas regiones por otras y a establecer estrategias de ayuda al desarroIlo que, enmarcadas en el ámbito de su política exterior, responden a lógicas propias. Y aunque la configuración de estas economías emergentes como nuevos donantes desconoce en cierta manera la participación de algunos de estos países en la elaboración de políticas de cooperación al desarroIlo años atrás (por ejemplo, la construcción de líneas de ferrocarril en África durante la década de 1960 gracias a la cooperación de China, o la participación de India como país donante en Nepal durante la década de 1950) ${ }^{4}$, cabe señalar que el paso dado por estos países desde economías meramente dependientes de ayuda a economías donantes de ayuda las convierte en economías que re-emergen, impulsadas por condiciones propias de su modelo de desarrollo, permitiéndoles así establecer sus propios lineamientos de políticas de ayuda al desarrollo.

\footnotetext{
2 El Grupo de los 20 ó G20 fue creado en 1999 en Berlín para atraer a los países industrializados y economías emergentes con el fin de discutir asuntos claves de la economía global. Entre sus miembros están: Argentina, Australia, Brasil, Canadá, China, Francia, Alemania, India, Indonesia, Italia, Japón, México, Rusia, Arabia Saudita, Sudáfrica, Korea del Sur, Turquía, Reino Unido y Estados Unidos. Ver: https://www.g20.org/ about_what_is_g20.aspx. Última consulta, abril 1 de 2008.

${ }^{3}$ Cfr. Sanauja, 2007. P. 47.

${ }^{4}$ Cfr. Swiss Agency for Cooperation and Development, 2007. P. 1
} 
Como señala Richard Manning, del Instituto de Desarrollo Exterior (ODI, por sus siglas en inglés): «deberíamos reconocer que la mayoría de los donantes que se encuentran fuera del CAD tienen una historia como donantes y muchos de ellos una buena experiencia. Deberíamos, por tanto, prestar atención no sólo a sus políticas de ayuda, sino al impacto general que puedan tener en los países pobres ${ }^{5} . »$

Teniendo en cuenta esta realidad, puede hablarse de tres categorías de países. La primera, los países miembros de la $\mathrm{OCDE}^{6}$ que no pertenecen al CAD, como la República Checa, Korea, MéxiCo, Turquía, Hungría, Islandia, Polonia y Eslovaquia. La segunda, los países de Oriente Medio y de la Organización de los Países Exportadores de Petróleo -OPEC-7 que proporcionan asistencia a los países musulmanes y países de África. La tercera, los países que no pertenecen a la OCDE, como Brasil, Rusia, la India y China, conocidos como BRIC y que son considerados por los propios integrantes del CAD como economías emergentes y miembros potenciales de la organización.

Esta última categoría, sobre la cual se centrará el presente análisis, ha sido el blanco hacia el cual los miembros del CAD han decidido dirigir el diálogo y los esfuerzos de coordinación en materia de ayuda al desarrollo. Esto debido a que su condición de economías emergentes les ha permitido desarrollar sus propias políticas y elaborar estrategias en materia de ayuda, especialmente en el continente africano. Así, la importancia estratégica que representan los países africanos para las economías emergentes en términos de recursos y que los ha llevado a dirigir sus políticas de ayuda hacia África, así como la urgencia de responder a las necesidades de esta región en el marco de los Objetivos del Milenio -ODM ${ }^{8}$, ha motivado a los donantes tradicionales -es decir, los miembros del CAD- a dirigir su atención hacia este grupo de países.

\footnotetext{
${ }^{5}$ Manning, 2006. P. 1.

${ }^{6}$ La OCDE es una organización internacional que agrupa a los 30 países económicamente más fuertes del mundo. Sus principales miembros son: Australia, Austria, Bélgica, Canadá, República Checa, Dinamarca, Finlandia, Francia, Alemania, Grecia, Hungría, Islandia, Irlanda, Italia, Japón, Korea, Luxemburgo, México, Países Bajos, Nueva Zelanda, Noruega, Polonia, Portugal, Eslovaquia, España, Suecia, Suiza, Turquía, Reino Unido, Estados Unidos. Ver: Organization for European Economic Co-Operation (OECD) «Members and partners» En: http://www.oecd.org/pages/0,3417,en_36734052_36761800_1_1_1_1_1,00.html. Última consulta, abril 2 de 2008.

${ }^{7}$ La OPEC es una organización permanente e intergubernamental creada en la Conferencia de Bagdad, en septiembre 10-14 de 1960. Su principal objetivo es coordinar y unificar las políticas petroleras para garantizar precios justos y estables de los productores. Sus principales miembros son: Argelia, Angola, Ecuador, Irán, Iraq, Kuwait, Libia, Nigeria, Qatar, Arabia Saudí, Venezuela, Emiratos Árabes. Ver: Organization of the Petroleum Exporting Countries (OPEC). «Brief History.» En: http://www.opec.org/aboutus/history/history.htm. Última consulta, abril 4 de 2008.

${ }^{8}$ Según el informe sobre los Objetivos del Desarrollo del Milenio de Naciones Unidas de 2008, el continente africano aparece como una de las regiones prioritarias en materia de desarrollo. Ver: Naciones Unidas. Objetivos de Desarrollo del Milenio. Informe 2008. En: http://www.un.org/spanish/millenniumgoals/pdf/ MDG_Report_2008_SPANISH.pdf. Última consulta, abril 6 de 2008.
}

EL SURGIMIENTO DE NUEVOS DONANTES Y SUS IMPLICACIONES PARA LA ARQUITECTURA DE LA AYUDA AL DESARROLLO 


\subsection{La cooperación de China como primer ejemplo}

China es un claro ejemplo de economía emergente que ha dado un salto cualitativo desde una economía dependiente de ayuda al desarrollo hacia una economía que coopera con países de África, principalmente. Esto lo clasifica como nuevo donante no miembro del CAD, pero con potencial para ser incluido dentro de la OECD?

El caso de China es especial porque ha suscitado posiciones a favor y en contra en lo referente a su política de cooperación al desarrollo. Siendo una economía emergente que tradicionalmente ha dependido de la propia ayuda al desarrollo, ha pasado en las últimas décadas a convertirse en una fuerte economía que ayuda a los más pobres. Su presupuesto de ayuda al desarrollo -aunque no ha sido publicado aún- se estima en 5 billones de dólares al año -según la Unión Europea-, a diferencia de India y Rusia que contribuyen con 100 millones de dólares, cada uno, al año ${ }^{10}$.

De este presupuesto de ayuda al desarrollo para finales del 2006, el gobierno de Beijing anunció que para 2009 duplicaría la asistencia en África, región prioritaria de su ayuda, proporcionando préstamos y créditos para los africanos y estableciendo un fondo de desarrollo que aliente a las compañías chinas a que inviertan allí. Asimismo, como parte de esta ayuda se contempla la cancelación de deuda, el entrenamiento de unos 15.000 africanos, la asignación de becas, el envío de expertos en temas agrícolas, la construcción de escuelas y hospitales, la asignación de medicamentos y el establecimiento de acuerdos de zonas económicas, entre otros ${ }^{11}$.

La política de ayuda al desarrollo de China como país donante no miembro del CAD y cuya economía depende fuertemente de recursos minerales (gas y petróleo), responde sin duda a una estrategia basada en desarrollar aquellas áreas que pueden servir a los intereses nacionales. Así por ejemplo, la Reunión Anual del Banco de Desarrollo Africano -AFDB, por sus siglas en inglés- puso sobre la mesa el interés de China sobre la región, al mismo tiempo que evidenció las grandes posibilidades de que este país se convirtiera en un modelo de desarrollo para África. Frente a este hecho, el Presidente de Madagascar Marc Ravalomanana, refiriéndose a China, aseguró que «Ustedes (China) son un ejemplo de transformación; nosotros en África debemos aprender de su éxito» ${ }^{12}$.

\footnotetext{
${ }^{9}$ Con respecto a países como Brasil, China, India y Sudáfrica, la OECD ha manifestado su interés por lograr que hagan parte de la organización. Fue así como en mayo de 2007 los países de la OECD acordaron invitar a países como Chile, Estonia, Israel, Rusia y Eslovenia para dialogar sobre su posible membrecía y ofrecer la posibilidad de incluir a Brasil, China, India, Indonesia y Sudáfrica a la organización. No obstante, al parecer estos últimos no han mostrado interés alguno en convertirse en miembros de la OECD. Cfr. The OECD, Organization for Economic Co-operation and Development. (2008) «The OECD: What is it?» En: www.oecd.org. Última consulta, abril 10 de 2008.

${ }^{10}$ Deutsches Institut fur Entwicklungspolitik (DIE), 2007. P. 1.

${ }^{11}$ Cammack, 2006. P. 1.

${ }^{12}$ Bezlova, 2007.
} 
No sólo es la primera vez que la reunión anual del Banco se celebra en Asia, sino la segunda vez que se hace por fuera de África. El Inter Press Service de Johanesburg calificó a China como un poder económico que está principalmente interesado en alcanzar sus propios intereses. Podría asegurase, por tanto, que no fue una casualidad que la reunión se llevara a cabo en China, país que ve en la región una oportunidad para beneficiarse a través de la adjudicación de ayuda al desarrollo de los recursos y del potencial económico que representa el continente. En este sentido, la cooperación al desarrollo dada por China se constituye en un instrumento para el logro de sus intereses en aquellas zonas de importancia en términos de energía, minerales y materiales crudos. Tal es el caso de Zimbabwe, Angola, Nigeria y Sudán que, debido a su riqueza en recursos naturales y minerales, representan más del $35 \%$ de la inversión directa extranjera de China en la región ${ }^{13}$.

Por tanto, el interés de China es convertirse en el principal y más importante inversor y proveedor de ayuda al desarrollo en África, a tal punto que le permita acceder más fácilmente a los recursos que necesita para alimentar su propia economía. Como señala Jonathan Holslag, investigador del Instituto de Bruselas de estudios de Chino contemporáneo -BICCS, por sus siglas en inglés-:

«Países como Brasil, Rusia, india y China están a la cabeza a la hora de establecer rápidamente nuevas relaciones económicas con África. Sin embargo, no todo son buenas noticias pues su apetito por recursos minerales no necesariamente se traduce en la creación de nuevos puestos de trabajo, lo que podría incrementar problemas de seguridad en África» ${ }^{14}$.

Asimismo, su política de cooperación al desarrollo se caracteriza por no condicionar la ayuda a cuestiones políticas, ni siquiera en aquellos países con bajos niveles de gobernanza. En este sentido, no discrimina entre regímenes corruptos y autoritarios para realizar la ayuda en aquellos países en los que su importancia en términos de recursos minerales así como mercados potenciales los convierte en zonas prioritarias en materia de cooperación. Este hecho podría, según el CAD, minar los esfuerzos logrados por la comunidad donante para establecer acuerdos universales de estándares de gobernanza con los países africanos. Esto en la medida en que el fácil acceso a la ayuda de China que desconoce condicionantes podría generar un sentimiento de irresponsabilidad en los gobiernos que cada vez tenderán a privilegiar la cooperación sin condicionantes en términos políticos, posponiendo de esta manera sus obligaciones en materia de gobernabilidad y buenas prácticas.

No obstante esta realidad, cabe preguntarse hasta qué punto los modelos de liberalización política y económica -que corresponden al establecimiento de democracias de corte occidental y economías de mercado- que condicionan la ayuda dada por los miembros del CAD a los países más pobres y que necesariamente deben cumplirse para poder obtener recursos de cooperación, se han traducido en una mejora a la situación de estos países.

Y aunque no se trata de decir que la ayuda tenga que darse de manera deliberada y sin compromisos, ya que podría suceder que los propios recursos fueran objeto de corrupción y malas prácticas

\footnotetext{
${ }^{13}$ Cfr. Op. Cit. Swiss agency for Cooperation and Development (SDC). 2007. P. 1.

${ }^{14}$ Holslag, 2008.

EL SURGIMIENTO DE NUEVOS DONANTES Y SUS IMPLICACIONES PARA LA ARQUITECTURA DE LAAYUDA AL DESARROLLO
} 
teniendo en cuenta que muchos de los países receptores de ayuda carecen de instituciones fuertes que garanticen un buen manejo de los mismos, lo que aquí se cuestiona es hasta dónde el modelo propiamente occidental y las medidas tomadas, como la implantación de democracias de tinte occidental y políticas de liberalización de mercados que condicionan la ayuda, son las más apropiadas para obtener resultados positivos en materia de gobernabilidad, reducción de la pobreza, paz y seguridad.

Como señala el Primer Ministro de Etiopía, Meles Zenawi:

«Las reformas neoliberales que han sido el modelo aplicado por occidente y el propio Banco Mundial en su intervención en África por más de dos décadas han fracasado en su intento por generar el tipo de crecimiento y desarrollo que ellos buscaban. Por tanto, creo que sería incorrecto que la gente en occidente asumiera que pueden comprar buena gobernabilidad en África. Lo que han hecho los chinos es explotar esa ilusión. China no pone de ninguna manera en peligro las reformas democráticas y aquellas encaminadas a una mejor gobernabilidad en África pues sólo aquéllas con raíces locales (o producidas localmente) tienen alguna posibilidad de éxito» ${ }^{15}$.

Es así como la ayuda ofrecida a África por nuevos donantes como China es vista desde otra perspectiva como un modelo que podría ayudar a los países pobres, debido a que su reciente éxito económico y continuo progreso al desarrollo los convierte en países que más acertadamente podrían entender la situación actual en la que se encuentran estos países, dado su paso reciente de economías en desarrollo a economías más avanzadas. Asimismo, porque además de aplicar estrategias de desarrollo que se salen de la ortodoxia occidental, como es el caso de Korea y Taiwan que han optado por una combinación entre instrumentos de mercado y de no mercado para optimizar su desarrollo, estas economías emergentes actualmente se encuentran involucradas en el proceso de avanzar cada vez más en términos de desarrollo, a diferencia de los donantes tradicionales cuyo paso al desarrollo fue logrado mucho tiempo atrás ${ }^{16}$.

Esta situación pone en evidencia que el surgimiento de nuevos donantes en el actual contexto internacional está cuestionando tanto la arquitectura como las políticas de ayuda al desarrollo que tradicionalmente han sido dirigidas y coordinadas por los países miembros del CAD. La emergencia de países como China pone al descubierto que existen otros actores que actuando por fuera del CAD también pueden contribuir a la ayuda al desarrollo, gracias a su condición de economías emergentes. Surge así la necesidad de un diálogo con los nuevos donantes para determinar hasta dónde la actual arquitectura y políticas en materia de ayuda -coordinada y dirigida por los países miembros del CAD- requiere de una revisión exhaustiva que permita abordar los nuevos desafíos que se ponen sobre la mesa en materia de ayuda. Al mismo tiempo, que permita reconocer que el surgimiento de nuevos donantes conlleva la aparición de nuevos valores y nuevas formas de abordar e interpretar la cooperación al desarrollo. Se cuestiona, por tanto, la legitimidad del CAD para gestionar las políticas de ayuda.

\footnotetext{
${ }^{15}$ Wallis, 2007.

${ }^{16}$ Op. Cit., Swiss agency for Cooperation and Development (SDC). 2007. P. 3
} 


\subsection{Brasil, India y Rusia: economías emergentes y su papel como nuevos donantes}

India, Brasil y Rusia son tres potencias emergentes que ejercen un papel destacado a nivel regional e internacional. No sólo poseen un gran potencial en recursos naturales y capacidades humanas -que les ha permitido ejercer un papel preponderante en el actual contexto internacional-, sino que son potencias emergentes con un peso demográfico y económico importante para constituirse en nuevos donantes de ayuda al desarrollo. Del mismo modo, estas tres potencias emergentes participan activamente en foros internacionales como la Organización de las Naciones Unidas, la Organización Mundial del Comercio -OMC-y la Cumbre del G-8, celebrada en Alemania en junio de $2007^{17}$.

\subsubsection{Brasil: entre África y Latinoamérica}

El caso de Brasil es bien interesante porque su importancia en los ámbitos político y económico le ha permitido jugar un rol preponderante a nivel regional con ambiciones de carácter global. Su reciente categoría se ve reflejada en cuestiones como su reclamo por un asiento permanente en el Consejo de Seguridad de Naciones Unidas, así como en el rol de liderazgo que ejerce en el G20, situación que lo convierte en nuevo donante de ayuda al desarrollo.

Y aunque el impacto de su ayuda dista mucho del impacto de la ayuda proporcionada por China en cuanto al volumen de fondos que este último puede proveer-, su rol como poder emergente comprometido con la cooperación al desarrollo de los más pobres ha demostrado ser un elemento decisivo en lo que se refiere a «...modelar la arquitectura de la gobernabilidad global, en tanto que Brasil ha ganado el reconocimiento y la confianza del Sur, porque se percibe a sí mismo como un intermediario entre los débiles y los fuertes ${ }^{18}$. Así, por ejemplo, el Ministro Federal Alemán califica a Brasil como un «un país ancla» con el cual los países miembros del CAD podrían hacer uso de su calidad como catalizador.

En lo que respecta a su política de cooperación al desarrollo, Brasil se caracteriza por no condicionar su ayuda a criterios políticos -como los exigidos por los miembros de la CAD a los países receptores de ayuda- que condicionan la cooperación al desarrollo a criterios de gobernanza o protección de los derechos humanos. Su cooperación se realiza, por tanto, sin ligamentos de tipo político con países considerados de alta prioridad, según sus intereses nacionales y su política exterior. Así como en el caso de China, con Brasil se cuestiona una vez más el enfoque político adoptado por los miembros del CAD de condicionar la ayuda a asuntos de gobernabilidad y buenas prácticas, lo que de alguna manera evidencia las diferentes maneras como los nuevos donantes abordan la ayuda al desarrollo.

Al igual que China, la cooperación de Brasil está dirigida principalmente hacia África, aunque no hay que desconocer el papel clave que tiene en Latinoamerica. Por ejemplo, en su carácter de

\footnotetext{
${ }^{17}$ Merkel, 2007.

${ }^{18}$ Schlager, 2007. P. 2

EL SURGIMIENTO DE NUEVOS DONANTES Y SUS IMPLICACIONES PARA LA ARQUITECTURA DE LA AYUDA AL DESARROLLO
} 
observador en el Club de París, Brasil ha participado en algunos casos en medidas de alivio de la deuda a favor de países africanos. Esto ha sido posible gracias a que es percibido como el tercer país emergente acreedor más grande, después de China y de Kuwait, que otorga préstamos a países de bajos ingresos ${ }^{19}$. Asimismo, sus vínculos culturales con África son considerados como una ventaja comparativa en términos de su relación con esta región que no sólo le ha permitido acercarse a países como Angola, Cabo Verde, Mozambique, Santo Tomé y Príncipe, sino que le ha ayudado a desarrollar una política de cooperación al desarrollo más consciente con las necesidades de estos países.

Un claro ejemplo de ello fue la celebración en noviembre de 2006, y por iniciativa del Presidente brasileño, de la Primera Cumbre África-Sudamericana con el propósito de fortalecer la cooperación y democratizar las relaciones internacionales, así como de reformar el Consejo de Seguridad de Naciones Unidas. Este hecho no sólo le permitió a Brasil poner en evidencia su liderazgo como nuevo donante, sino que le aseguró de alguna manera el apoyo de las naciones africanas para obtener un asiento permanente en dicho órgano ${ }^{20}$.

En términos de montos de ayuda, se calcula que el auxilio proveniente de Brasil supone 436 millones de dólares proporcionados por el Banco Nacional de Desarrollo Económico y Social -BNDESpara proyectos de infraestructura en Angola. Estos proyectos. que se estima durarán 9 años. fueron iniciados en 2007 por la Compañía de Construcción Brasilera Odebrecht, que proporcionará tratamientos de aguas residuales, alumbrado público y construcción de caminos en los principales poblados de la provincia de Benguela, en Angola ${ }^{21}$.

Por otro lado, Brasil representa para Latinoamérica un gran potencial en términos de desarrollo, paz y estabilidad regional. Con 8.514.876,599 km², que corresponde a la mitad del continente americano, y con una población de 185 millones de habitantes, que representan un tercio de la población latinoamericana, Brasil es una potencia de cooperación que puede contribuir en términos políticos y económicos. Así, por ejemplo, el gobierno brasilero ha mediado en conflictos políticos, como el , de Bolivia, al igual que ha participado en la coordinación del diálogo entre el gobierno colombiano y las guerrilla de las FARC, así como en la entrega de secuestrados por parte de esta guerrilla. Estas acciones, que son de alguna manera muestra del interés de Brasil por lograr la estabilidad en la región, ponen en evidencia el liderazgo del país en el conjunto de Latinoamérica.

En términos económicos, Brasil cuenta con un gran potencial en energías renovables al tiempo que es considerado como el país con mayor experiencia en la producción de etanol y biodisel ${ }^{22}$. Este

${ }^{19}$ Cfr. Ibíd., p. 4.

${ }^{20}$ Cfr. Ibíd., p. 5.

${ }^{21}$ Cfr. International Water and Sanitation Centre (IRC). 2006. «Emerging donors: Chinese and Brazilian Involvement in the Water Sector in Africa.» En: http://www.irc.nl/page/31876. Última consulta, abril 15 de 2008.

22 De Sousa, 2007. P. 37. 
hecho se constituye en una gran ventaja para la región en términos de protección del medioambiente, al igual que en términos de inversión, en tanto que la región se constituye en un gran potencial en el sector de energías renovables que la ubican en una posición de liderazgo en este tema. Asimismo, Brasil ha liderado iniciativas importantes de política internacional como la lucha contra la pobreza y el hambre en el mundo. Esta iniciativa fue lanzada por el presidente brasilero Lula Da Silva en el foro Action Against Hunger and Poverty, en 2004, y contó con el apoyo de países como Francia, Chile y España, así como del Secretario General de Naciones Unidas de ese entonces, Kofi Annan $^{23}$. Este tipo de iniciativas son muestra del marcado dinamismo de su política exterior que lo configuran como una economía emergente con gran potencial de ayuda.

\subsubsection{La India y su potencial como nuevo donante de ayuda}

El caso de la India es también importante, pues al igual que China tiene cierta experiencia como país donante, particularmente durante la década de 1950 con Nepal. Con un PIB que llega a los 785 mil millones de dólares y un gasto militar que supera dos veces el de sus vecinos, durante los últimos años India no sólo se ha convertido en una de las principales promotoras de la integración económica regional, al incentivar el desarrollo de la Asociación Sudasiática para la Cooperación Regional -SAARC, por sus siglas en inglés ${ }^{24}$, sino que se ha constituido en una economía emergente con potencial para constituirse como nuevo donante de ayuda para cooperar con los países más pobres.

Su política de ayuda al desarrollo combina una variedad de instrumentos de asistencia y entrenamiento técnico, al igual que de ayuda concesional. Su política exterior se ha caracterizado por su participación activa en foros internacionales. Así, por ejemplo, durante la Guerra Fría el país desempeñó un importante papel al interior del Movimiento de los No Alineados $-\mathrm{MNA}^{25}$, en donde se propuso dar un mayor peso a los países en vías de desarrollo. Por otro lado, India se constituye en un gran contribuyente a las misiones de mantenimiento de la paz de Naciones Unidas, lo que responde de alguna manera a su interés por lograr un asiento permanente en el Consejo de Seguridad de este organismo.

\footnotetext{
${ }^{23}$ Para mayor información, ver: http://www.globalpolicy.org/socecon/glotax/general/2004/0920newyork.pdf. última consulta, abril 10 de 2008.

${ }^{24}$ Creada en diciembre de 1985 con el propósito de proveer a sus miembros una plataforma para el trabajo conjunto con un espíritu de amistad, confianza y entendimiento. Apunta a acelerar el proceso de desarrollo económico y social de sus miembros, entre los que se encuentran: Bangladesh, Bután, India, Maldivas, Nepal, Paquistán y Sri Lanka. Ver: http://www.saarc-sec.org/. Última consulta, abril 11 de 2008.

${ }^{25}$ Creado en la Conferencia de Belgrado en 1961 por los presidentes de Egipto, India y Yugoslavia, el MNA se define a partir de una serie de principios entre los que se encuentran: preservar las independencias nacionales frente a las dos superpotencias; no pertenecer a ningún bloque militar; rechazar el establecimiento de bases militares extranjeras; defender el derecho de los pueblos a la autodeterminación y la independencia; y luchar por un «desarme completo y general». Ver. Historia del siglo XX. «Movimiento de los países no alineados». En: http://www.historiasiglo20.org/GLOS/noalineados.htm. Última consulta, abril 12 de 2008.
} 
La Iniciativa de Desarrollo de la India, aún a la espera de ser aprobada, pone énfasis en un nuevo instrumento financiero por medio del cual el país pretende conceder préstamos de bajo coste a países de África Subsahariana. Asimismo, la India espera aumentar su contribución en África en alrededor de 300-400 millones de dólares, 10 veces más de la contribución hecha en el período 2004-2005 ${ }^{26}$. Para el Consejo de Inteligencia Nacional de Estados Unidos, la India se convertirá en 2025 en la cuarta economía más grande del planeta ${ }^{27}$.

Al igual que China, India ofrece como parte de su política de cooperación ayuda a regiones de África que son ricas en recursos naturales y minerales. Poco dispuesto a alinear sus políticas de cooperación con los estándares dispuestos por el CAD, India -al igual que Brasil y China- ha optado por desarrollar sus propias políticas de ayuda en el marco de su política exterior, en aqueIlas regiones que representan un verdadero interés para su economía.

Junto con Brasil y Sudáfrica, India se comprometió en 2003 como economía emergente a realizar una alianza para aumentar el impacto de estas tres economías en el escenario global, al mismo tiempo que a fomentar la cooperación. Este hecho se formalizó en el Foro de Diálogo IBSA -India, Brasil y Sudáfrica- en el que se suscribieron acuerdos de cooperación regional y global. Posteriormente, en septiembre de 2006 los jefes de gobierno de los tres países participaron en la Primera Cumbre IBSA en Brasilia, en donde coordinaron sus posiciones para la 61 Asamblea General de Naciones Unidas en la que Sudáfrica fue elegida como miembro no permanente del Consejo de Seguridad, poniendo en evidencia una vez más la importancia tanto política como económica de estas tres economías emergentes.

Con respecto a Sudáfrica, aunque en términos económicos, territoriales y demográficos el país no tiene un peso significativo con respecto a sus vecinos, en términos democráticos y de estabilidad el país cuenta con gran potencial para promover el desarrollo democrático y el libre mercado en el resto del continente. Esto se debe en gran parte a que su política exterior le ha dado una importancia significativa a los asuntos africanos y en especial a la relación con sus vecinos. Así, por ejemplo, Sudáfrica participa en la Nueva Alianza para el Desarrollo del Sur de África -NEPAD, por sus siglas en inglés- en donde representa el 76\% del PIB de la región ${ }^{28}$. Asimismo, Sudáfrica, a la cabeza de su presidente Thabo Mbeki, ha sido promotor de la Unión Africana -UA- y del New Partnership for Africa's Development-NEPAD-, que junto con otros estados africanos busca combatir la pobreza y el subdesarrollo del continente ${ }^{29}$.

\footnotetext{
${ }^{26}$ Cfr. Op. Cit., Manning, 2006. P. 6.

27 Daniel, 2007. P. 32.

${ }^{28}$ GELB, 2002. P. 14.

${ }^{29}$ Para mayor información, ver: http://www.nepad.org/. Última consulta, abril 15 de 2008.
} 


\subsubsection{Rusia y su conquista de África}

Como las demás economías emergentes, Rusia es un actor clave en materia de ayuda al desarrollo gracias al acelerado crecimiento de su economía. Como donante de ayuda al desarrollo, para los próximos cuatro años se calcula que Rusia contribuirá con un total de 250 millones de dólares en esta materia. Asimismo, durante los últimos cinco años Rusia ha asistido a los países afectados por emergencias humanitarias con un total de 55 millones de dólares, como es el caso de Angola, Irán, Paquistán, Líbano, Argelia, Angola, Mali, Sudán, Cuba, Bolivia, México y Ecuador ${ }^{30}$.

En 2007, Rusia aprobó su estrategia de ayuda que apunta a incrementar sus contribuciones en esta materia. Con un total de 400-500 millones de dólares por año, el gobierno ruso, junto con el Banco Mundial -BM- y la Organización Mundial de la Salud -OMS-, busca atender las necesidades humanas y de desarrollo, especialmente en África y Asia central ${ }^{31}$. En palabras del Ministro de Finanzas de Rusia: «Rusia está expandiendo su contribución de ayuda al desarrollo, particularmente en el control de enfermedades infecciosas, educación y acceso a energía» ${ }^{32}$.

La contribución de Rusia a programas de ayuda en África evidencia por un lado su rol protagónico como nuevo donante de ayuda y como economía emergente en el actual contexto internacional. Por otro lado, demuestra el interés de Rusia en el continente africano como una zona estratégica de recursos minerales. Por ello, Rusia ha comenzado a ingresar paulatinamente en África, especialmente por medio de contratos de gas y petróleo, con el fin de ganar mayor influencia en el mercado mundial de energía. Por ejemplo, en septiembre del año anterior Rusia logró concesiones de gas en Nigeria, considerada una de las regiones con mayores reservas mundiales en este recurso, a través de su monopolio energético Gazprom ${ }^{33}$.

De la mano de esta iniciativa, Rusia le prometió al continente africano ayuda al desarrollo materializada en la producción de electricidad, así como en la construcción de un gasoducto transahariano de $4.000 \mathrm{~km}$ hacia Europa. Asimismo, Rusia ha firmado acuerdos en esta materia con países como Argelia, Angola, Egipto y Costa de Marfil. Por otro lado, Rusia ha participado de programas de cancelación de la deuda en África por 11,3 billones de dólares, incluyendo \$2,2 billones de deuda perteneciente a los países pobres fuertemente endeudados, por medio de la iniciativa Heavily Indebted Poor Countries - HIPC, por sus siglas en inglés $-{ }^{34}$.

\footnotetext{
30 Alimov, A.

${ }^{31}$ Vasilieva, 2007. "Russia Contributes US\$20 Million to Malaria Control in Africa». The World Bank. En: http://go.worldbank.org/8CYJJ1N2Y0. Última consulta, abril 16 de 2008.

32 Ibíd.

${ }^{33}$ «Rusia trata de conquistar África». Revista Foreign Policy en español. Especial web. En: http://www.fpes.org/rusia-trata-de-conquistar-africa. Última consulta, abril 15 de 2008.

${ }^{34}$ La iniciativa HIPC o Heavily Indebted Poor Countries fue creada en 1996 por el BM y FMI con el propósito de proveer a los países más pobres y fuertemente endeudados un alivio a la deuda. Ver: World Bank. «(HIPC) The Enhanced Heavily Indebted Poor Countries Initiative». En: http://go.worldbank.org/85B908KVE0. Última consulta, abril 16 de 2008.
} 
No obstante, al igual que China, Brasil e India, la ayuda rusa se caracteriza por no estar condicionada ni ligada a elementos de carácter político. Esto, en últimas se traduce en una cooperación de tipo incondicional que no se limita por factores democráticos o buenas prácticas.

\section{POSIBLES RIESGOS Y BENEFICIOS PARA LA AYUDA AL DESARROLLO A PARTIR DE LA EMERGENCIA DE NUEVOS DONANTES NO MIEMBROS DEL CAD}

Si bien es cierto que el debate en torno a los posibles riesgos y beneficios que conlleva la cooperación dada por los nuevos donantes o economías emergentes, como India, China, Brasil y Rusia, ha suscitado posiciones encontradas, es evidente que estos aspectos evidencian que la arquitectura y las políticas de desarrollo están siendo cuestionadas a tal punto que resulta imprescindible analizar los futuros desafíos que la actual ayuda al desarrollo deberá enfrentar, a partir del surgimiento de nuevos donantes no miembros del CAD.

EI CAD, como único foro en donde los países e instituciones miembros se reúnen para incrementar la efectividad y coordinar la política de ayuda al desarrollo, se constituye en el mayor donante de ayuda al desarrollo que busca por medio de esa ayuda «...que los países en desarrollo tengan la capacidad de participar en la economía global y sobreponerse a la pobreza ${ }^{35}$. Creado en 1961, ha servido como plataforma clave para discutir cuestiones referentes a la ayuda. Así, por ejemplo, con respecto a la emergencia de nuevos donantes, el 6 y 7 de abril de 2006 se llevó a cabo la Conferencia de donantes emergentes en el desarrollo global comunitario.

Bajo la Presidencia de Rusia en el G8 y con la colaboración de la OCDE y el Banco Mundial, la Conferencia reconoció la importancia de la contribución a nivel global de los nuevos donantes a la ayuda al desarrollo, resaltando el papel preponderante que podrían jugar al asistir a países menos desarrollados en el logro de los ODM. Asimismo, la Conferencia instó a los nuevos donantes y a los miembros del CAD a desarrollar un diálogo directo en temas de ayuda, con el propósito de aunar fuerzas para lograr un mayor impacto en las necesidades puntuales de regiones prioritarias como África ${ }^{36}$.

Es así como se reconoce, en primer lugar, que los nuevos donantes pueden contribuir con nuevas perspectivas y nuevos procesos de ayuda al desarrollo, para la configuración de la agenda de desarrollo global. Esto es posible gracias a que estos países por medio de su ayuda proporcionan nuevos valores y nuevas oportunidades de cooperación. Al mismo tiempo, presentan un amplio margen de estrategias de desarrollo y prácticas que son de gran valor para la arquitectura de cooperación al

${ }^{35}$ Ibíd.., p. 18.

${ }^{36}$ Cfr. Emerging Donors in the Global Development Community. International Conference hosted by the Russian G8 Presidency Moscow, 6 - 7 April 2006. President Hotel, Moscow, Russian Federation. En: http:// www.oecd.org/dataoecd/34/56/36417981.pdf. Última consulta, abril 16 de 2008. 
desarrollo, que pueden contribuir no sólo en términos económicos, sino en términos estratégicos y políticos a reorientar y perfilar desde una nueva perspectiva la actual arquitectura de cooperación. Eckhard Deutscher, el nuevo Director del CAD, afirma al respecto:

«tenemos que estar muy abiertos a lo que los nuevos donantes pueden aportar (...) China, por ejemplo, puede aprender mucho de nuestra experiencia, al igual que nosotros podemos aprender de la experiencia de China. (...)Tenemos que continuar escuchando, pues aunque tenemos gran experiencia de décadas pasadas, el escenario global ha cambiado» ${ }^{37}$.

En segundo lugar, teniendo en cuenta que la ayuda al desarrollo de los principales donantes del mundo cayó en 2007 un 8,4\%, la participación de los nuevos donantes en materia de ayuda, especialmente en los países de África, puede verse como un ejemplo de cooperación y un llamado de atención a los países miembros de la OCDE para que asuman mayores compromisos con los países que necesitan la ayuda. Y aunque la Declaración de París 2005 es un intento por mejorar la calidad de la ayuda y su impacto en el desarrollo, así como de promover un modelo basado en la apropiación, alineamiento, armonización, gestión por resultados y mutua rendición de cuentas $\mathrm{s}^{38}$, urge la necesidad de que estos compromisos se traduzcan en resultados puntuales, como el incremento de la ayuda. Así, los nuevos donantes se constituyen en una nueva fuente de contribución en materia de cooperación que puede incrementar la ayuda al desarrollo. Como señala Vivien Foster, economista del Banco Mundial y experta en desarrollo en África, «las necesidades de África van más allá de lo que lo donantes tradicionales de ayuda oficial al desarrollo-AOD- son capaces de proveen ${ }^{39}$.

En tercer lugar, la configuración de las economías emergentes en nuevos donantes se traduce en mayores ventajas comparativas para la cooperación al desarrollo. Estos países han ganado un gran peso a nivel regional e internacional que les ha permitido incrementar la cooperación en sus regiones, gracias a la cercanía geográfica y cultural. Al mismo tiempo, se convierten en países intermediarios que debido a su experiencia y conocimiento de la zona pueden profundizar y desarrollar proyectos de cooperación de manera más acertada. Brasil es un claro ejemplo de ello al ser considerado por el Ministerio Federal Alemán de Cooperación Económica y Desarrollo-BMZ- como un país «ancla» que le permitirá profundizar y desarrollar estrategias con los donantes tradicionales, especialmente en el ámbito regional gracias a su importancia y liderazgo en este nivel.

En cuarto lugar, lo nuevos donantes, que han dado el paso desde economías puramente dependientes de ayuda a economías donantes, se constituyen en modelos de desarrollo que pueden aportar

\footnotetext{
${ }^{37}$ Jaura, R. 2008. "Q\&A: Head of Aid Committee calls for Partnership with China.» Global Information Network. New York: Mar 18, 2008. En: http://ins.onlinedemocracy.cal index.php?name $=$ News\&file $=$ article \&sid $=11480$. Última consulta, abril 16 de 2008.

${ }^{38}$ Cfr. Development Co-operation Directorate (DCD-DAC). «The Paris Declaration.» February 28. March 2005. En: http://www.oecd.org/dataoecd/11/41/34428351.pdf. Última consulta, abril 20 de 2008.

${ }^{39}$ Inagaki, K. 2008. «G8 seeks emerging donors' help on development.» Kyodo News. En: http:// search.japantimes.co.jp/cgi-bin/nn20080402f1.html. Última consulta, abril 22 de 2008.
} 
nuevas estrategias y promover políticas de ayuda en los países más pobres a partir de su propia experiencia pasada que les ha permitido avanzar hacia el progreso. De alguna manera, su paso reciente de un modelo en desarrollo a un modelo más avanzado les da la ventaja de poder comprender la situación actual de muchos países dependientes de ayuda, y los convierte en modelos ejemplares de crecimiento y progreso.

Asimismo, pueden ayudar a aumentar la capacidad de negociación de regiones como África, al constituirse en nuevas fuentes de ayuda. Por tanto, su experiencia puede contribuir al entendimiento y elaboración de políticas de ayuda más acertadas que se traduzcan en mejoras para los países más necesitados. Como señala el Ministro de Asuntos Exteriores de Japón Masahiko Komura: «queremos que los nuevos donantes compartan su propia experiencia de desarrollo con los países especialmente de África que continúan siendo pobres» ${ }^{40}$.

Teniendo en cuenta algunos de los posibles beneficios de la participación de los nuevos donantes en el actual contexto internacional de ayuda al desarrollo, podría decirse que estos nuevos actores tienen la capacidad de hacer sus propias contribuciones en esta materia, en la medida en que además de estar comprometidos con la ayuda al desarrollo en regiones prioritarias como África, también han adoptado estrategias propias y nuevas perspectivas a la hora de abordar la ayuda al desarrollo. Tanto los valores que permean sus políticas de ayuda como su experiencia a la hora de abordar el tema del desarrollo pueden contribuir a la transformación de la actual arquitectura y de la política de cooperación. Así, por ejemplo, se afirma que «los participantes nuevos aportan aire fresco, recursos, innovación, herramientas nuevas y naturalmente algo de competencia, lo que en principio debería conducir a una reducción de costos y una mejor entrega de la asistencia $»^{41}$.

No obstante, al tiempo que estos nuevos actores de la cooperación podrían contribuir al debate en torno a la ayuda, también podrían representar un factor de riesgo para la actual arquitectura de cooperación. Existe la preocupación, en primer lugar, de que las estrategias y políticas de cooperación empleadas por los nuevos donantes $-y$ que se caracterizan por la ayuda no ligada a criterios de gobernabilidad y buenas prácticas- puedan de alguna manera minar los esfuerzos realizados por los donantes tradicionales para mejorar estos aspectos. Esto en la medida en que el acceso a la ayuda no condicionada podría generar desincentivos en los gobiernos receptores de la ayuda para realizar reformas de gobierno, al igual que para adoptar buenas prácticas en la gestión de los recursos.

En segundo lugar, la ayuda proveniente de los nuevos donantes podría traducirse en una situación de insostenibilidad de la deuda de los países más pobres, porque la proliferación de préstamos

${ }^{40}$ Shimizu, K. 2008. «G8, emerging donors start development talks in Tokyo.» En: http://search.japantimes.co.jp/ cgi-bin/nn20080406a3.html. Última consulta, abril 25 de 2008.

${ }^{41}$ Kaberuka, D. 2008. «La nueva arquitectura de la asistencia internacional: nuevos participantes, nuevos participantes, nuevos desafíos, ¿viejos problemas?» Ideas para el Desarrollo. En: http:// www.ideas4development.org/la-nueva-arquitectura-de-la-asistencia-internacional-nuevos-participantes-nuevosdesafios-\%c2\%bfviejos-problemas/es/. última consulta, abril 26 de 2008. 
baratos y de millones de dólares en ayuda incondicional podría volver a ubicar a estos países en una situación de deuda insostenible, de la que recientemente muchos han salido gracias a los programas de alivio de la deuda. Como señala Hilary Benn, Secretaria de Desarrollo Internacional de Gran Bretaña, «el dinero chino puede producir más daño que beneficio. Si los países están tomando préstamos en gran cantidad hasta el punto de que su deuda llegue a ser insostenible, esto minará todo el trabajo que se ha hecho para enfrentar el problema de la deuda. La cuestión de la deuda no es la deuda per se, sino si ésta se puede soportar $»^{42}$.

En tercer lugar, la participación de los nuevos donantes podría traducirse en una proliferación incontrolada de programas y proyectos en regiones como África -zona prioritaria de cooperación tanto para los donantes tradicionales como para los nuevos donantes-, pues no existen marcos de coordinación y diálogo en los que puedan enmarcarse las distintas estrategias e intereses de los donantes en esta región. Por tanto, más que un incremento en la calidad de la ayuda al desarrollo, podría darse un incremento de la cantidad, que no necesariamente se traduciría en mejores políticas para la atención de necesidades puntuales en los países receptores de ayuda.

Como resultado, los nuevos donantes, respondiendo a sus propias estrategias y políticas de cooperación así como a sus intereses puntuales, terminarían privilegiando sus propios programas y proyectos, desconociendo tal vez la existencia de programas similares. Esto conllevaría la réplica de esfuerzos en materia de cooperación así como el desperdicio en recursos materiales y humanos. Según lo anterior, existe una urgencia por lograr una mayor coordinación y coherencia con estos nuevos actores de la ayuda al desarrollo.

En cuarto lugar, la falta de una estrategia clara de sistematización de la ayuda al desarrollo proveniente de los nuevos donantes ha impedido establecer el impacto real de dicha ayuda, lo que ha llevado a desconocer la importancia y el potencial en materia de cooperación que pueden aportar estos nuevos actores en esta materia. Asimismo, este hecho complejiza aún más la coordinación de los donantes tradicionales con los nuevos donantes, porque al no existir estadísticas reales de la ayuda proporcionada por estos países resulta más difícil llegar a acuerdos reales y estrategias puntuales en el campo de la ayuda al desarrollo. Por tanto, se tiende a hablar erróneamente de la ayuda proveniente de estos países así como a realizar estimaciones equívocas sobre el tipo de ayuda.

\section{FUTUROS DESAFÍOS PARA LA ARQUITECTURA DE AYUDA AL DESARROLLO A PARTIR DE LA EMERGENCIA DE NUEVOS DONANTES NO MIEMBROS DEL CAD}

Con el propósito de lograr los ODM, los países signatarios de la Declaración de París reconocieron la importancia de incrementar la cantidad y la calidad de la ayuda al desarrollo. Asimismo, identi-

\footnotetext{
42 "Chinese Aid to Africa may do more harm than good, warns Benn.» The Guardian. En: http://
} www.guardian.co.uk/world/2007/feb/08/development.topstories3. Última consulta, abril 28 de 2008.

EL SURGIMIENTO DE NUEVOS DONANTES Y SUS IMPLICACIONES PARA LA ARQUITECTURA DE LAAYUDA AL DESARROLLO 
ficaron la necesidad de llevar a cabo una mayor coordinación entre los países donantes y los países receptores de la ayuda, con el fin de entablar un diálogo más acertado sobre esta ayuda. En este sentido, uno de los grandes desafíos a partir de este compromiso tiene que ver, en primer lugar, con la capacidad de los donantes tradicionales de armonizar sus políticas de cooperación con los nuevos donantes que, actuando por fuera del CAD, tienden a privilegiar sus propias estrategias y políticas de ayuda al desarrollo, motivados por sus propios intereses.

Por tanto, los donantes tradicionales comprometidos con las obligaciones adquiridas en la Conferencia de París, al mismo tiempo que conscientes de los nuevos desafíos que comporta la emergencia de nuevos donantes para la actual arquitectura y política de ayuda al desarrollo, deberían desarrollar un diálogo constructivo con los nuevos donantes, considerando la importancia y la urgencia de responder a los ODM y a las necesidades puntuales de regiones prioritarias como África.

Como parte de este diálogo constructivo, es importante, en segundo lugar, que se reconozcan las contribuciones que los nuevos donantes puedan hacer en materia de ayuda al desarrollo. Al mismo tiempo que sean considerados los nuevos mecanismos y estrategias de cooperación empleados por estos países, con el propósito de aprender de sus propios procesos de desarrollo así como de sus estrategias de ayuda. Es importante dejar claro que los esfuerzos en materia de ayuda deben estar dirigidos a asistir a los países menos desarrollados en el logro de las metas del milenio, por lo que cualquier contribución y lectura alternativa proporcionada por los nuevos donantes encaminada a este fin debería ser contemplada como una oportunidad para redefinir y reorientar la actual política de cooperación, hacia una política de ayuda más efectiva.

En tercer lugar, otro gran desafío tiene que ver con la necesidad de coordinar esfuerzos e intereses entre los donantes con el propósito de lograr establecer estrategias puntuales de ayuda que impidan la réplica de esfuerzos, al igual que la pérdida de recursos materiales y humanos. Así por ejemplo, teniendo en cuenta que África es considerada como una región prioritaria en materia de cooperación, sería importante lograr que tanto los donantes tradicionales como los nuevos donantes coordinaran políticas y estrategias de ayuda para asistir de manera más acertada las necesidades puntuales de esta región.

En cuarto lugar, es indispensable que se logre una sistematización más eficaz de la ayuda proveniente de los nuevos donantes, ya que la ausencia de sistematización impide establecer la magnitud e impacto que podría tener la ayuda de los nuevos actores. Por tanto, otro gran desafío tiene que ver con la necesidad de armonizar los sistemas estadísticos de los donantes, para poder determinar la cuantía, los sectores y las regiones hacia donde va dirigida la ayuda al desarrollo de los nuevos donantes.

Finalmente, es importante que los países miembros del CAD -donantes tradicionales de ayuda al desarrollo- sean conscientes de la nueva realidad que actualmente caracteriza el actual contexto de cooperación y de la necesidad de adaptar las antiguas políticas y estrategias de ayuda al nuevo panorama en el que la emergencia de los nuevos donantes cuestiona la pertinencia de la actual 
arquitectura de ayuda al desarrollo, demandando así una redefinición y adaptación más acertada de la estrategia de cooperación a las nuevas realidades cambiantes.

\section{CONCLUSIÓN}

Es definitivo y cierto que la arquitectura actual de ayuda al desarrollo está cambiando. La emergencia de nuevos donantes pone sobre la mesa la necesidad de adecuar la estructura vigente de cooperación al desarrollo a la nueva realidad y a los nuevos desafíos que comporta la participación de estos nuevos actores en materia de ayuda. La emergencia de nuevos donantes además de contribuir a la política de ayuda misma, por medio de los aportes que ellos puedan proporcionar, implica una serie de riesgos que deberán ser tenidos en cuenta en aras de lograr un mejor impacto y mayores beneficios para los países objeto de ayuda. La necesidad de lograr un diálogo constructivo y de coordinar esfuerzos con los nuevos donantes es uno de los principales desafíos que deben enfrentar los donantes tradicionales con el propósito de incrementar la efectividad y la calidad de la ayuda.

En el marco de este diálogo deberían reconocerse las diferencias entre los actores de la cooperación, armonizar sus estrategias de ayuda y llegar a un acuerdo entre las partes que permita considerar que más allá de la diferencia entre nuevos y viejos donantes existe una realidad puntual que demanda una mayor coordinación y adopción de marcos comunes. Además, resulta necesario establecer una visión compartida que permita concluir de que sólo a través de los esfuerzos colectivos puede contribuirse de manera más eficaz al problema de la pobreza en el mundo.

\section{BIBLIOGRAFÍA}

Alimov, A. «Re-emerging as donor. Rusia's participation in global development cooperation.» Department of International Organizations, Ministry of Foreign Affairs of the Russian Federation. Presentación Power Point, (sf).

Bezlova, A. 2007. «China as a Role Model for Development». Johanesburg, Inter Press Service.

Cammack, D. 2006. «China and the politics of development in Africa». Overseas Development Institute ODI-.

Daniel W. 2007.«The New World Order». Foreign Affairs, marzo-abril.

Deutsches Institut Fur Entwicklungspolitik -DIE-. 2007. «Trilateral Development Cooperation with 'New Donors'». Briefing Paper No. 5.

Emerging Donors in the Global Development Community. International Conference hosted by the Russian G8 Presidency Moscow, 6-7 April 2006. President Hotel, Moscow Russian Federation. En: http://www.oecd.org/ dataoecd/34/56/36417981.pdf.

Development Co-operation Directorate -DCD-DAC-. «DAC Members and date of membership» En: http:// www.oecd.org/document/38/0,3343,en_2649_34603_1893350_1_1_1_1,00.html 
Development Co-operation Directorate-DCD-DAC-. 2005. «The Paris Declaration». En: http://www.oecd.org/ dataoecd/11/41/34428351.pdf.

De Sousa, S. 2007. «La India, el Brasil y Sudáfrica: ¿potencias emergentes o países en desarrollo? Potencias regionales y poderes emergentes: hacia una reconfiguración del orden global». Revista Iberoamericana de Análisis Político.

Gelb, S. 2002. «South Africa and the New Partnership for Africa's Development». The Edge Institute. En: www.the-edge.org.za/pdf/sanepad.pdf.

Historia del siglo XX. «Movimiento de los Países no Alineados». En: http://www.historiasiglo20.org/GLOS/ noalineados.htm.

Holslag, J. «The new scramble for Africa». Europe's World, the only European-wide policy journal. En: http:// www.europesworld.org/EWSettings/Article/tabid/78/Default.aspx?ld =16d5d99d-ad2e-43f6-a51c-1dad3a0cfe82.

Inagaki, K. 2008. "G8 seeks emerging donors' help on development». Kyodo News. En: http:// search.japantimes.co.jp/cgi-bin/nn20080402f1.html

International Water and Sanitation Centre -IRC-. «Emerging donors: Chinese and Brazilian involvement in the water sector in Africa». Diciembre 2006. En: http://www.irc.nl/page/31876

Jaura, R. 2008. "Q\&A: Head of Aid Committee calls for Partnership with China». Global Information Network. New York. En: http://ins.onlinedemocracy.ca/index.php?name $=$ News\&file $=$ article $\&$ sid $=11480$

Kaberuka, D. 2007. «La nueva arquitectura de la asistencia internacional: nuevos participantes, nuevos desafíos, ¿viejos problemas?». Ideas para el desarrollo. En: http://www.ideas4development.org/la-nueva-arquitectura-dela-asistencia-internacional-nuevos-participantes-nuevos-desafios-\%c2\%bfviejos-problemas/es/

Manning, R. 2006. «Will «Emerging Donors» Change the Face of International Cooperation?». Overseas Development Institute-ODI-.

Merkel, A. 2007. «G-8 for Sharing Global Responsibility With Emerging Economies - Angela Merkel». South Bulletin.

Naciones Unidas. Objetivos de desarrollo del milenio. Informe 2008. En: http://www.un.org/spanish/ millenniumgoals/pdf/MDG_Report_2008_SPANISH.pdf

Organization for European Economic Co-operation -OECD-. «Members and partners». En: http://www.oecd.org/ pages/0,3417,en_36734052_36761800_1_1_1_1_1,00.html

Organization of the Petroleum Exporting Countries -OPEC-. «Brief History.» En: http://www.opec.org/aboutus/ history/history.htm

«Rusia trata de conquistar África». Foreign Policy. Especial web. En: http://www.fp-es.org/rusia-trata-de-conquistar-africa

Sanauja, J. 2007. « ¿Un mundo unipolar, multipolar o apolar? El poder estructural y las transfor5maciones de la sociedad internacional contemporánea». Curso de Derecho Internacional y Relaciones Internacionales de Vitoria. Gasteiz. 
Schlager, C. 2007. «iNew Powers for Global Change? Challenges for International Cooperation: the case of Brasil». Dialogue on Globalization. Briefing Pappers. Berlin, Friedrich Ebert Stiftung -FES-

Shimizu, K. 2008. «C8, emerging donors start development talks in Tokyo.» En: http://search.japantimes.co.jp/ cgi-bin/nn20080406a3.html

Swiss Agency for Cooperation and Development-SDC-. 2007. «New donors: China's Africa policy as a prime example». Federal Department of Foreign Affairs -FDFFA-. Development Policy Paper.

Vasilieva, M. 2007. «Russia Contributes US\$20 Million to Malaria Control in Africa». The World Bank. En: http://go.worldbank.org/8CYJJ1N2Y0. Última consulta, abril 16 de 2008.

World Bank. «HIPC. The Enhanced Heavily Indebted Poor Countries Initiative». En: http://go.worldbank.org/ 85B908KVE0 